\title{
Effect of static foot posture on the dynamic stiffness of foot joints during walking
}

\section{Word count: 2997}

\section{Abstract}

This study aimed to analyse the dynamic stiffness of foot joints during gait in the sagittal plane in feet with different static foot postures. Seventy healthy adult male subjects with different static postures, assessed by the Foot Posture Index (FPI) (30 normal, 20 highly pronated and 20 highly supinated), were recruited. Kinematic and kinetic data were recorded using an optical motion capture system and a pressure platform, and dynamic stiffness at the different stages of the stance was calculated from the slopes of the linear regression on the flexion moment-angle curves. The effect of foot type on dynamic stiffness and on ranges of motion and moments was analysed using ANOVAs and post-hoc tests, and linear correlation between dynamic stiffness and FPI was also tested. Highly pronated feet showed a significantly smaller range of motion at the ankle and metatarsophalangeal joints and also a larger range of moments at the metatarsophalangeal joint than highly supinated feet. Dynamic stiffness during propulsion was significantly greater at all foot joints for highly pronated feet, with positive significant correlations, although small, with the squared FPI. Highly supinated feet showed greater dynamic stiffness than normal feet, although to a lesser extent. Highly pronated feet during normal gait experienced greatest decrease in the dorsiflexor moments during propulsion. Normal feet were found to be the most balanced regarding work generated and absorbed.

\section{Keywords}

Dynamic Stiffness; foot joints; Foot Posture Index; range of motion; walking.

\section{Highlights}

- All foot types undergo approximately constant dynamic stiffness in the same phases

- Dynamic stiffness during propulsion at all joints differs with foot type

- Pronated feet experience greatest dorsiflexor moment decrease during propulsion

- Highly pronated feet have to absorb more work, having a higher risk of damage

- Highly supinated feet present intermediate dynamic stiffness and absorbed work 


\section{Introduction}

Foot injuries, such as hallux valgus or plantar fasciitis (prevalences $37 \%$ and $7 \%$, respectively [1]) are related to abnormal joint motion, but more relevantly to abnormal forces [2]. Analysis of the foot joints dynamics during gait can help understanding the development of these injuries [3]. Different works undertook this analysis by looking at the dynamic joint stiffness [4], [5], defined as the ratio between the external moment applied to the joint and the joint angle, at a specific time, assessed while performing activities that require muscle activation, such as walking. This stiffness combines the effect of muscle forces, inertia and deformation of soft tissue, and was already applied to the ankle in the sagittal plane with different purposes [4], [6], [7]. High and low dynamic stiffness have been related with a higher incidence of bone injuries [8], [9] and with excessive joint motion and less joint stability [10] respectively. Besides, the analysis of the dynamic stiffness is also valuable for providing mechanical properties of the foot joints to be used when designing orthotics or prostheses, and also to check the effect of surgeries that may modify joint stiffness.

Recently, the authors analysed the flexion stiffness of the ankle, midtarsal and metatarsophalangeal joints during normal walking in healthy normal subjects [11], identifying different stance phases in which moment and angle changes were linearly related, i.e. with an approximately constant dynamic stiffness: early and late midstance phases and propulsion phase at the ankle and midtarsal joints, and propulsion phase at the metatarsophalangeal joint. The study of these dynamic stiffnesses in feet with different static postures may help to understand the well-known relationship between the static foot posture and the development of lower limb injuries [12]. To date, only the effect of the static posture on foot kinematics during gait has been studied [13]-[16], but reporting contradictory data. These works found inconsistent data in peak value dependency, probably because they are affected by the reference posture [12], [14], but also by the approach applied to determine relative motion. They also reported different results regarding range of motion (ROM). While some studies observed a decreased ankle ROM of pronated feet in the sagittal plane [15], [16] and increased in the frontal plane [15], [17], [18], other works found no significant differences in any motion plane [19], [20]. At the midtarsal joint, one study observed a decrease in the ROM for pronated feet in the transverse plane [13], although others found no significant differences in any motion plane [19]. And at the 
metatarsophalangeal joint one study observed a reduction in the ROM in the sagittal plane for pronated feet [15].

Differences in ROM results among works may be due also to differences in the static foot posture index used in each study, and to differences in the samples (age, sex, etc.). There are currently different methods available for quantifying the static foot posture [21], the foot posture index (FPI) being reported to be more reliable than other indices to estimate the foot dynamic function [22], [23]. As the analysis of the effect of the FPI on the foot joints dynamics has been limited to their kinematics and reported contradictory data, this study aimed to analyse the effect of FPI on the foot dynamics in the principal plane of motion, the sagittal plane, during normal gait. The analysis included the comparison of the ROM, the moment ranges and the dynamic stiffnesses throughout the stance phase, and their relationship with FPI.

\section{Material and methods}

\section{Experiment description}

The study was carried out on 70 adult male subjects without a history of neuromuscular problems, diabetes or foot or ankle surgery, and who did not use orthotics nor reported pain in the lower extremity. The subjects were recruited with normal (from 0 to +5 ), highly pronated (HP) (higher than +10 ) or highly supinated (HS) (lower than -5) static FPI on both feet, as measured by Redmond et al. [22] (descriptive data in Table 1), all participants presenting very similar FPI values in both feet. All of them provided written informed consent to participate in the study, which was approved by the ethical committee of the Universitat Jaume I (Castellón, Spain).

The subjects were asked to walk barefoot along a 7-m walkway at a comfortable self-selected speed, stepping with their right foot on a pressure platform located in the middle of the walkway. Before data collection, the subjects were familiarized with the conditions by walking on the walkway several times. The subjects had to look forwards while walking, to avoid platform targeting, and they repeated the activity as many times as needed to have five valid trials, trials where they did not step on the platform with the right foot being discarded.

\section{Data acquisition}


The dynamics of the ankle, midtarsal and metatarsophalangeal joints of the right foot were registered using an adaptation of the model proposed by Bruening et al. [24], as presented in Sanchis-Sales et al. [11]. This model considered the midtarsal and metatarsophalangeal joints globally, not one particular midtarsal or metatarsophalangeal joint.

Segment position and orientation were tracked at a $100 \mathrm{~Hz}$ sampling rate by an eight infrared camera motion analysis system (Vicon Motion Systems Ltd., Oxford, UK). Joint angles were calculated, from the upright standing static reference posture, using a Cardan rotation sequence between distal and proximal segments: 1-dorsiflexion/plantarflexion (DF/PF), 2-abduction/adduction (AB/AD), and 3inversion/eversion (IN/EV) [25]. All kinematic data were low-pass filtered with a $4^{\text {th }}$-order Butterworth filter and cut-off frequency of $10 \mathrm{~Hz}$.

Contact pressures of the right foot were recorded at a $100 \mathrm{~Hz}$ sampling rate with a Podoprint pressure platform (Namrol Group, Barcelona, Spain) synchronized with the infrared camera system. In each frame, pressure data were segmented by comparing the contact-cell coordinates with the anteroposterior location of the joint centres for the time when the foot was fully contacting on the platform (E.g., cells with anteroposterior-coordinate between those of midtarsal and metatarsophalangeal joint centres were assigned to the forefoot segment). The normal component of the ground reaction forces and centre of pressure (CoPs) were calculated on each foot segment (taking into account the contact cells area), and joint moments in the sagittal plane were then calculated from them and expressed relative to the orientation of the local coordinate system of the proximal segment. Calculated joint moments were normalized to body-weight, consistently with previous publications [4], [5], and were low-pass filtered with a $4^{\text {th }}$-order Butterworth filter and cut-off frequency of $50 \mathrm{~Hz}$.

\section{Dynamic stiffness calculation}

As in a previous work [11], dynamic stiffnesses were computed as the slopes of the linear regressions at those phases where the dorsiflexion moment-angle relationship was approximately linear (Figure 1): early midstance, and propulsion for the ankle $\left(K_{\text {ankle }}^{E M S P}\right.$ and $\left.K_{\text {ankle }}^{P P}\right)$, late midstance and propulsion for the midtarsal joint $\left(K_{M T}^{L M S P}\right.$ and $\left.K_{M T}^{P P}\right)$, and propulsion for the metatarsophalangeal joint $\left(K_{M P}^{P P}\right)$.

Phases were trimmed by $5 \%$ at both the onset and ending of each phase, and then the dynamic stiffness was calculated as the slope of the linear regression of the joint moment versus the joint angle, 
i.e. the tangent of the angle from the horizontal to the interpolated straight line. However, the tangent function is non-linear and presents a discontinuity at $90^{\circ}$, which may introduce errors when calculating mean values and when applying ANOVAs. To avoid these problems, mean calculations and ANOVAs were performed directly on the angles $(\theta)$, and results were finally transformed into dynamic stiffness data by computing the tangent of the angle data.

\section{Statistical analysis}

For each foot type, and in each foot joint, plots with the means and 95\% confidence intervals (CI) were presented for the dorsiflexion angle and moment along the stance phase from all the trials and subjects. And mean joint moments were plotted versus mean joint angles, along with the linear regressions representing the dynamic stiffnesses in each of the above-mentioned phases.

For each subject, the ROMs, the ranges of joint moments and the angles representing the dynamic stiffness in each phase were averaged across the five trials recorded, as in a previous work [11]. Three sets of ANOVAs were performed to check for the effect of foot type, considering statistical significance at 0.05 level: i) one ANOVA on the ROM with foot type as factor (normal, HP or HS) in each joint; ii) one ANOVA per joint on the range of joint moments with foot type as factor, in each joint; and iii) a set of ANOVAs (one for each phase at each joint) on the angles representing the dynamic stiffness as the dependent variable, with foot type as factor. Tukey post-hoc tests were performed for a deeper understanding when significant differences were detected. Finally, Pearson's correlations between dynamic stiffnesses and FPI and squared FPI were also calculated.

\section{Results}

The plots of the joint dorsiflexion moments versus the joint dorsiflexion angles during the stance phase (Figure 1) showed a counterclockwise loop at the ankle for the normal FPI feet, in agreement with previous works [6], [11], and a clockwise loop for both HP and HS feet, enclosing less area in the case of normal FPI feet. At the midtarsal and metatarsophalangeal joints, all loops were clockwise, with normal FPI feet also enclosing less area at the midtarsal joint. The lowest linearity in the phases studied were for propulsion at the metatarsophalangeal joint, although R2 values were still above 0.90 . And the dynamic stiffness was greater for HP feet during propulsion, in all joints. 
The curve profiles for the joint dorsiflexion angle and moment along the stance phase (Figures 2 and 3) were similar for all foot types, but with differences in the peak values and timings during the propulsion, which resulted in the differences in the dynamic stiffnesses mentioned above.

Insert Figure 2 here

Insert Figure 3 here

For each foot type, statistical data for the ROM and range of moments at all joints, together with the dynamic stiffness in the different phases considered, are reported in Table 1, and significant statistical differences can be observed in Table 2 . HP feet had a significantly smaller ROM than the other foot types considered at both the ankle and metatarsophalangeal joints. Likewise, they were subjected to a significantly larger range of moments than HS feet at the midtarsal joint, while HS feet were subjected to a significantly smaller range of moments at the metatarsophalangeal joint than the other foot types. At the ankle and midtarsal joints, the dynamic stiffness was significantly different for each foot type considered during propulsion, with the greatest values for HP feet, followed by HS feet. At the metatarsophalangeal joint the same trend could be observed, but in this case significant differences were found only between HP feet and the other feet. No significant differences were observed for the dynamic stiffness in any phase of the stance period other than the propulsion phase in any of the foot joints.

\section{Insert Table 1 here}

Insert Table 2 here

Finally, positive significant correlations, although small, were found between $K_{a n k l e}^{P P}, K_{M T}^{P P}$ and $K_{M P}^{P P}$, and squared FPI ( $r=0.614,0.623$ and 0.647 , respectively).

\section{Discussion}

This work aimed to study the differences in the dynamic stiffnesses of the foot joints during normal gait in the sagittal plane for feet with different static foot postures. Novelty values of the dynamic stiffnesses were provided for normal, HP and HS feet, which could help better comprehend the functioning of the different foot joints during gait for these foot types, but also may help surgeons quantify the mechanical effect of their operations, and in the design of foot prostheses and orthotics. 
The dorsiflexion moment-angle curves presented in this study agreed with those from previous works [5], [11], having identified similar phases with approximately linear moment-angle behaviour for all foot types considered. During the stance phase the foot joints presented a variable dynamic stiffness, in agreement with the classical interpretation of the foot as a mobile adapter during the initial contact allowing adjustment to varying terrain and as a rigid lever for forward propulsion in locomotion [2], [26]. The metatarsophalangeal joint presents less periods with constant dynamic stiffness, with much smaller values than those of the ankle or midtarsal joints during propulsion. Although approximately constant dynamic stiffness were found for all foot types considered at the same phases, the specific values differed among them.

Before analysing the differences in the dynamic stiffnesses with the static foot posture, it is advisable to first look at the variation of joint angle and moment during the stance phase. A significant smaller ROM was found for HP feet in both ankle and metatarsophalangeal joints, accordingly to previous works [15], [16]. These differences were due basically to the differences in the evolution of the dorsiflexion angles during propulsion (Figure 2). The ROM used during the propulsion phase was smaller in HP feet for all foot joints considered, with significantly reduced final plantarflexion angle, which might be related with the shorter length of the triceps surae muscle reported in HP feet [26], [27], which could be studied in future works. Conversely, the dorsiflexion angle curves for HS feet during the stance phase were quite close to those of normal FPI feet. Dorsiflexion moment curves during the stance phase (Figure 3) followed a bell-shaped profile in all foot types considered, with similar peak values except for the metatarsophalangeal joint. Moment ranges (which corresponded approximately to moment peak values) were significantly larger at the midtarsal joint in HP feet when compared to HS feet (although differences were small), and were significantly smaller at the metatarsophalangeal joint in HS feet. Pronation seemed to generate larger peak values of dorsiflexion moment, especially at the metatarsophalangeal joint, favoured by a larger distance between the COP and the joint centres (about 1.5 times that of the HS feet) because of a smaller dorsiflexion joint angle at this time. This might explain the higher incidence of hallux limitus in HP feet [28].

Angles and moments data at each joint were combined in the dorsiflexion moment-angle curves. At the ankle, a counterclockwise loop was observed in the moment-angle curve for normal FPI feet, 
revealing a positive net work supplied by the joint internal forces: the area beneath the rising phase of the loop (midstance) represents the work absorbed mainly by joint passive structures, with some contribution of plantarflexor muscles to stabilise the joint; while the area beneath the descending phase (propulsion) represents the work produced by the internal forces, essentially muscular plantarflexor forces (under concentric contraction), although some contribution also arises from the energy stored and released in passive tissues during walking. This loop became clockwise for HP and HS feet, with the largest area (negative net work) for HP feet. At the midtarsal and metatarsophalangeal joints the loop was in the clockwise direction for all foot types considered, enclosing very different areas depending on the foot type in the case of the midtarsal joint, with the largest negative work corresponding to HP feet. Normal FPI feet presented the smallest areas within the loops at the ankle and midtarsal joints, i.e. these feet were the best balanced regarding the difference between the work generated and absorbed, while passive tissues in HP feet during midstance had to absorb more work than that required for propulsion, consequently being more demanded and subjected to a higher risk of damage [29]. In practice, the orthoses for HP and HS feet should be designed to favour a more balanced net work.

Focusing on the dynamic stiffness in the phases considered, significant differences were found during propulsion in all foot joints, with the largest values in HP feet, and the normal FPI feet being the ones with the smallest values. This was consistent with the differences found in the ROM, and could be related with the decreased concentric plantarflexion strength of HP feet at the ankle, when compared to normals, reported in literature [30]. During propulsion the plantarflexor muscles were subjected to concentric contraction, so that their decreased strength became into a more pronounced decrease of the moment they generate than that observed in normal FPI feet. This effect was usually presented in literature as a loss of rigidity in pronated feet, in the sense that plantarflexor muscles generate less torque on the joint [30]. This expression should be avoided, as in fact the dynamic stiffness is greater in absolute terms, i.e. there is a greater change (decrease) in the moment generated by plantarflexor muscles during propulsion in HP feet. Therefore, the greater dynamic stiffness of HP feet during propulsion compared to normal FPI feet reflected a poorer capacity for generating force, therefore generating joint instability which increases the joint sprain risk factor. These differences might play a 
more important role in other situations where propulsion gains importance, such as running or climbing stairs. A greater dynamic stiffness was also observed in HS feet when compared to normal feet, although to a lesser extent, consistently with the smaller differences observed both in the joint angles and joint moments.

This study presented some limitations and results should be taken cautiously. A greater number of subjects could shed light on the influence of static foot posture on dynamic stiffness during the early and late midstance phases. The results are limited to the description of the mechanical behaviour of adult male subjects, and further research should focus on children, adolescents or the elderly. Also, it might be interesting to study the effect of the foot type in women, as they are especially susceptible to changes in joint flexibility [31]. The data reported for midtarsal and metatarsophalangeal joints does not allow the analysis of a particular joint. And finally, the tangential components of the ground reaction force were neglected. Anyway, their effect on the flexion moments is small because their magnitudes are much smaller than those of the normal component [32] and also the moment arms, as we checked (results not shown for brevity), so that the moment graphs reported here for the normal FPI sample are very close to those reported in previous works [33] that took into account the tangential components.

\section{Conclusion}

Novelty values of the dynamic stiffnesses during normal gait in the sagittal plane were provided for normal, HP and HS feet. All foot types experienced approximately constant dynamic stiffness during the same phases, but the values differed during propulsion. HP feet showed greatest dynamic stiffness values and absorbed work, followed by HS feet, which may generate further problems. The data presented could be of interest in further studies aimed at analysing the relationship between hallux limitus and the appearance of pathologies such as hallux abductus valgus or plantar fasciitis [34] or to determine the effect of surgery that modifies joint stiffness, like subtalar arthrodesis or arthrodesis of the first metatarsocuneiform joint, as well as plantar fasciotomy.

\section{Conflict of interest statement}

Conflicts of interest: none. 


\section{References}

[1] J. E. Dunn, C. L. Link, D. T. Felson, M. G. Crincoli, J. J. Keysor, and J. B. McKinlay, "Prevalence of Food and Ankle Conditions in a Multiethnic Community Sample of Older Adults," American Journal of Epidemiology, vol. 159, no. 5. pp. 491-498, 2004.

[2] K. A. Kirby, "Biomechanics of the normal and abnormal foot," J. Am. Podiatr. Med. Assoc., vol. 90, no. 1, pp. 30-4, Jan. 2000.

[3] A. D. Kuo and J. M. Donelan, "Dynamic principles of gait and their clinical implications.," Phys. Ther., vol. 90, no. 2, pp. 157-74, 2010.

[4] R. B. Davis and P. A. DeLuca, "Gait characterization via dynamic joint stiffness," Gait Posture, vol. 4, no. 3, pp. 224-231, 1996.

[5] K. Shamaei, G. S. Sawicki, and A. M. Dollar, "Estimation of Quasi-Stiffness and Propulsive Work of the Human Ankle in the Stance Phase of Walking," PLoS One, vol. 8, no. 3, 2013.

[6] H. Houdijk, H. C. Doets, M. van Middelkoop, and H. E. J. (Dirkjan) Veeger, "Joint stiffness of the ankle during walking after successful mobile-bearing total ankle replacement," Gait Posture, vol. 27, no. 1, pp. 115-119, 2008.

[7] Y. Sekiguchi, T. Muraki, Y. Kuramatsu, Y. Furusawa, and S.-I. Izumi, "The contribution of quasi-joint stiffness of the ankle joint to gait in patients with hemiparesis," Clin. Biomech., vol. 27, no. 5, pp. 495-499, 2012.

[8] D. S. Williams, I. S. McClay, and J. Hamill, "Arch structure and injury patterns in runners," Clin. Biomech., vol. 16, no. 4, pp. 341-347, 2001.

[9] D. S. Williams, I. M. Davis, J. P. Scholz, J. Hamill, and T. S. Buchanan, "High-arched runners exhibit increased leg stiffness compared to low-arched runners.," Gait Posture, vol. 19, no. 3, pp. 263-9, Jun. 2004.

[10] K. P. Granata, D. A. Padua, and S. E. Wilson, "Gender differences in active musculoskeletal stiffness. Part II. Quantification of leg stiffness during functional hopping tasks," $J$. Electromyogr. Kinesiol., vol. 12, no. 2, pp. 127-135, 2002.

[11] E. Sanchis-Sales, J. L. Sancho-Bru, A. Roda-Sales, and J. Pascual-Huerta, "Dynamic Flexion Stiffness of Foot Joints During Walking.," J. Am. Podiatr. Med. Assoc., vol. 106, no. 1, pp. $37-$ 
46, 2016.

[12] A. K. Buldt, G. S. Murley, P. Levinger, H. B. Menz, C. J. Nester, and K. B. Landorf, “Are clinical measures of foot posture and mobility associated with foot kinematics when walking?," J. Foot Ankle Res., vol. 8, p. 63, 2015.

[13] A. E. Hunt and R. M. Smith, "Mechanics and control of the flat versus normal foot during the stance phase of walking," Clin. Biomech., vol. 19, no. 4, pp. 391-397, 2004.

[14] P. Levinger and W. Gilleard, "Relationship between static posture and rearfoot motion during walking in patellofemoral pain syndrome: effect of a reference posture for gait analysis.," $J$. Am. Podiatr. Med. Assoc., vol. 96, no. 4, pp. 323-9, 2006.

[15] P. Saraswat, B. a. MacWilliams, R. B. Davis, and J. L. D'Astous, "Kinematics and kinetics of normal and planovalgus feet during walking," Gait Posture, vol. 39, no. 1, pp. 339-345, 2014.

[16] A. K. Buldt, P. Levinger, G. S. Murley, H. B. Menz, C. J. Nester, and K. B. Landorf, "Foot posture is associated with kinematics of the foot during gait: A comparison of normal, planus and cavus feet," Gait Posture, vol. 42, no. 1, pp. 42-48, 2015.

[17] A. E. Hunt, A. J. Fahey, and R. M. Smith, "Static measures of calcaneal deviation and arch angle as predictors of rearfoot motion during walking.," Aust. J. Physiother., vol. 46, no. 1, pp. 9-16, 2000.

[18] S. C. Cobb, L. L. Tis, J. T. Johnson, Y. T. Wang, M. D. Geil, and F. a. McCarty, "The effect of low-mobile foot posture on multi-segment medial foot model gait kinematics," Gait Posture, vol. 30, no. 3, pp. 334-339, 2009.

[19] D. W. Powell, B. Long, C. E. Milner, and S. Zhang, "Frontal plane multi-segment foot kinematics in high- and low-arched females during dynamic loading tasks," Hum. Mov. Sci., vol. 30, no. 1, pp. 105-114, 2011.

[20] C. J. Barton, P. Levinger, K. M. Crossley, K. E. Webster, and H. B. Menz, "Relationships between the Foot Posture Index and foot kinematics during gait in individuals with and without patellofemoral pain syndrome.," J. Foot Ankle Res., vol. 4, p. 10, Jan. 2011.

[21] M. Razeghi and M. E. Batt, "Foot type classification: a critical review of current methods.," Gait Posture, vol. 15, no. 3, pp. 282-291, 2002. 
[22] A. C. Redmond, J. Crosbie, and R. A. Ouvrier, "Development and validation of a novel rating system for scoring standing foot posture: The Foot Posture Index," Clin. Biomech. (Bristol, Avon), vol. 21, no. 1, pp. 89-98, 2006.

[23] R. G. Nielsen, M. S. Rathleff, C. M. Moelgaard, O. Simonsen, S. Kaalund, C. G. Olesen, F. B. Christensen, and U. G. Kersting, "Video based analysis of dynamic midfoot function and its relationship with Foot Posture Index scores,” Gait Posture, vol. 31, no. 1, pp. 126-130, 2010.

[24] D. A. Bruening, K. M. Cooney, and F. L. Buczek, “Analysis of a kinetic multi-segment foot model. Part I: Model repeatability and kinematic validity," Gait Posture, vol. 35, no. 4, pp. 529-534, 2012.

[25] E. S. Grood and W. J. Suntay, "A joint coordinate system for the clinical description of threedimensional motions: application to the knee.," J. Biomech. Eng., vol. 105, no. 2, pp. 136-44, 1983.

[26] K. A. Kirby, "Relatioship between Achilles tendon and plantar fascia tension," in Foot and Lower Extremity Biomechanics III: Precision Intricast Newsletters, Payson, AZ: Precision Intricast, 2008, pp. 100-101.

[27] J. Pascual Huerta, "The effect of the gastrocnemius on the plantar fascia.," Foot Ankle Clin., vol. 19, no. 4, pp. 701-18, Dec. 2014.

[28] K. a Kirby, "Subtalar joint axis location and rotational equilibrium theory of foot function.," $J$. Am. Podiatr. Med. Assoc., vol. 91, no. 9, pp. 465-487, 2001.

[29] Y. M. Golightly, M. T. Hannan, A. B. Dufour, H. J. Hillstrom, and J. M. Jordan, "Foot Disorders Associated with Over-Pronated and Over- Supinated Foot Function:The Johnston County Osteoarthritis Project HHS Public Access," Foot Ankle Int, vol. 35, no. 11, pp. 1159$1165,2014$.

[30] A. G. Snook, "The Relationship between Excessive Pronation as Measured by Navicular Drop and Isokinetic Strength of the Ankle Musculature," Foot Ankle Int., vol. 22, no. 3, pp. 234-240, 2001.

[31] M. Chiacchiero, B. Dresely, U. Silva, R. DeLosReyes, and B. Vorik, "The Relationship Between Range of Movement, Flexibility, and Balance in the Elderly," Top. Geriatr. Rehabil., 
vol. 26, no. 2, pp. 148-155, 2010.

[32] D. A. Bruening, K. M. Cooney, F. L. Buczek, and J. G. Richards, "Measured and estimated ground reaction forces for multi-segment foot models," J. Biomech., vol. 43, no. 16, pp. 32223226, 2010.

[33] D. A. Bruening, K. M. Cooney, and F. L. Buczek, "Analysis of a kinetic multi-segment foot model part II: Kinetics and clinical implications," Gait Posture, vol. 35, no. 4, pp. 535-540, 2012.

[34] Y. Aranda and P. V Munuera, "Plantar fasciitis and its relationship with hallux limitus.," J. Am. Podiatr. Med. Assoc., vol. 104, no. 3, pp. 263-8, 2014. 